\title{
PERSPECTIVES
}

OPINION

\section{Myelin inhibitors: does NO mean GO?}

\author{
Geoffrey Raisman
}

At least three molecules that are associated with oligodendrocytic myelin act through a common transduction mechanism that causes collapse of axonal growth cones in culture. It is generally considered that this mechanism underlies the failure of regeneration of cut axons in the adult mammalian central nervous system. In this article, I review evidence that these important findings have revealed, not an inhibitory mechanism, but a vital repulsive contact-guidance mechanism, which is necessary for the growth of adult axons along myelinated tracts in vivo.

A recent article by Filbin ${ }^{1}$ in this journal authoritatively reviewed the mounting evidence for a mechanism that causes axonal growth cones to collapse. In this model, at least three different molecules that are associated with oligodendrocytic myelin in the central nervous system (CNS) are linked by a common receptor mechanism to a signal transduction pathway, whose activation leads to growth cone collapse in tissue culture assays (see also REF. 2). The conclusion proposes that myelin-associated molecules are one of the principal causes of failure of regeneration of cut axons in the intact adult nervous system.

This view has been dominant since the early work of Schwab and colleagues ${ }^{3}$, and has led to the exploration of several new molecular strategies to induce regeneration in the adult brain and spinal cord by abrogating the effect of these proposed inhibitory molecules. If, however, the collapse of growth cones in culture does not reflect the mechanism for the inhibition of regeneration in vivo, these strategies would not be effective. Moreover, if, as I propose in this article, the collapse of growth cones in culture is actually a reflection of a mechanism that facilitates and accelerates regeneration in vivo, then such strategies might actually prevent regeneration.

\section{The myelin inhibitory hypothesis}

The original indication that central myelin inhibits axon growth came from a tissue culture assay in which it was shown that axons of cultured sympathetic or sensory neurons regenerate selectively on pieces of peripheral (sciatic) nerve, but avoid pieces of central (optic) nerve ${ }^{4}$. However, more recent observations show that the in vivo distribution of ligands and receptors that lead to the myelin inhibitory cascade in different fibre tracts in the central and peripheral nervous systems shows no correlation with the ability of individual tracts to regenerate ${ }^{5-8}$. Also, the proposal that central

\section{The proposal that myelin is} inhibitory to axon growth sits uncomfortably with the fact that myelinated fibre tracts are a widely conserved anatomical device through which diverse types of axons travel around the adult brain and spinal cord. myelin is inhibitory to axon growth does not explain why axons also fail to regenerate through grey matter, where there is no myelin. The proposal that myelin is inhibitory to axon growth sits uncomfortably with the fact that myelinated fibre tracts are a widely conserved anatomical device through which diverse types of axons travel around the adult brain and spinal cord. It is as if we were claiming that roads were inhibitory to the movement of cars, or rails to trains.

The myelin inhibitory hypothesis has been supported by reports that inhibition of specific myelin inhibitors promotes regeneration in vivo ${ }^{3}$. Apart from the use of antibodies, some suggested therapeutic approaches to overcome this inhibition are as extreme as immunizing animals against their own myelin ${ }^{9}$, a procedure that seems to be not only hazardous but counter-intuitive, as there is no evidence that patients with autoimmune demyelinating diseases, such as multiple sclerosis, can regenerate their spinal cords after injury. Attempts to show that genetic ablation of supposed myelin inhibitors will allow regeneration in adult mice have so far been inconclusive ${ }^{10-13}$.

On the other hand, several groups have reported that rapid and robust growth of both embryonic and adult axons occurs selectively along adult myelinated fibre tracts $^{14-18}$ (FIGS 1 and 2) and can lead to recovery of function ${ }^{19}$. The proponents of the myelin inhibitory theory have attempted to explain this apparent predilection of growing fibres for myelinated tracts in various ways. For example, it has been suggested that the axonal receptors for the inhibitory myelinassociated molecules might have been downregulated, or that the functional benefits are due to sprouting, not regeneration. In general, the attractiveness of a unitary molecular theory of inhibition with its apparent simplicity, and the economic promises for drug development, have been sufficient to overshadow any doubts arising from contrary observations. 


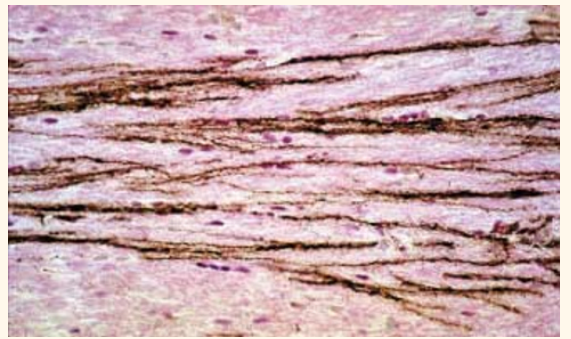

Figure 1 | Axon growth after neuronal transplantation. This picture shows a band of axons, immunostained with the M6 antibody, growing along the adult fimbria after transplantation of embryonic hippocampal neurons. These axons show the typical dispersion among the myelinated adult tract fibres ${ }^{17}$

\section{What are the alternatives?}

If myelin is not the cause of the disastrous failure of regeneration after brain and spinal cord injury, what is? After injury, adult axons sprout vigorously in the lesion area ${ }^{20}$ (FIG. 3a), but the sprouts remain restricted to the lesion site and cannot advance to their targets to restore functional connections. So, the idea that the failure of regeneration is due to something that prevents the advance of axon sprouts is attractive. However, it seems increasingly likely that this prevention of growth is associated not with oligodendrocytes and central myelin, but with reactive astrocytes $^{21-23}$. Astrocytes are present throughout the CNS - not only in grey matter, but also as an essential and universal structural feature of white matter tracts ${ }^{24}$ (FIG. 4a,b and Y. Li et al., unpublished data). In all sites, including the dorsal root entry zone (REF. 25 and Y. Li et al., unpublished data), they react sensitively to direct damage (FIG. 4c) or to the presence of axons degenerating as a result of a distant lesion. Prevention of axon advance by reactive astrocytes could therefore explain why regeneration fails in both grey and white matter. This might also explain the observations of structural and functional regeneration after transplantation of embryonic tissue ${ }^{26}$ or olfactory ensheathing cells, as these grafts can prevent or 'open up' astrocytic scars and form bridges across lesions ${ }^{27}$.

In contrast to the hypertrophic response of astrocytes to injury, oligodendrocytes are highly vulnerable ${ }^{28}$, and they respond to injury by rapid cell death ${ }^{29,30}$. Electron microscopy shows that dying oligodendrocytes and their associated myelin are promptly sequestered by phagocytic microglia and are removed, so they could not form a barrier to regeneration along denervated tracts. Our own analysis of axon growth through transplants of olfactory ensheathing cells in the severed optic nerve ${ }^{31}$ shows that regeneration fails when axons are confronted with an astroglial scar from which, as electron microscopy shows, all traces of oligodendrocytes and myelin have been removed completely.

What then is the explanation of the apparent paradox that myelin-associated molecules cause growth cone collapse in culture? As pointed out by Fawcett ${ }^{32}$, the tissue cultures that have been used to study this phenomenon are two-dimensional, and the simple confrontation of axons with myelin in culture does not reflect the complex arrangements in vivo. The main requirement for axon growth in vivo is that a growth cone elongates along a pathway. A parallel requirement is that it does not turn out of the tract and does not stop growing until it reaches its destination. When it reaches its destination, the axon leaves the tract and invades the target neuropil where it branches, usually profusely, to make terminal synaptic contacts. Branching is associated with the cessation of elongation ${ }^{33}$, and conversely, elongation is associated with the absence of branching. Inhibition of branching is an essential element in the assembly of long-distance connections in the growing CNS.

Perhaps the growth cone collapse that is observed when a front of axons extending across a tissue culture dish is faced with a homogeneous environment of myelinassociated molecules is an expression of the inhibition of branching that is needed for growth cone elongation in vivo. The effect of inhibition of branching in vivo might be to force the axons to continue, rather than to stop them. One might call this the 'skating rink' effect - the less the attraction between the skates and the ice, the faster and easier is the movement. A similar effect is exploited by high-speed trains that float over the rails, or hovercraft that glide on water.

The advance of a growing axon depends on its adhesive reaction to a positive and sustaining substrate. But the advancing tip of the axon is preceded by intense filopodial exploration. A single growth cone might produce a vast number of filopodia, which are intensely motile. By this means, the entire environment ahead is thoroughly and repeatedly scanned. The filopodia act as sensory antennae, whose principal, if not only purpose is to signal back to the growing axon which way not to go. We might regard the exploratory movements of the filopodia as analogous to the waving of the white stick of a blind man - he does not need to use the stick for advance or to aid walking; its purpose is to detect obstacles and prevent him from going in the wrong direction. In the developing optic pathways, for example, advancing growth cones are small, bullet-like structures without filopodia; where they pause at decision points, such as the optic chiasma, the growth cones expand and develop filopodia ${ }^{34}$. We note a similar difference between highly branched endings of adult corticospinal axons when they are blocked by a lesion (FIG. 3a), and the streamlined, small advancing tips of regenerating corticospinal axons when advancing through an olfactory ensheathing cell graft ${ }^{27}$ (FIG. 3b).

\section{Myelin as a facilitator}

An impressive accumulation of evidence $e^{35,36}$ indicates that the extraordinary accuracy with which developing axons are consistently guided to specific targets is achieved by the precise deployment of a combination of molecules, many of which can act at different times or places as both attractants and repellents to achieve an orderly pattern of growth ${ }^{37}$. Repulsion is at least as important an element in growth cone advance as facilitation. Axon behaviour in culture indicates that the default setting of an axon is to grow; advance is constitutive. But without the surround inhibition to provide repulsive contact guidance, the growth cones could not make the selection that is needed for them to choose their pathways.

The discovery of the repulsive molecules that are associated with the postnatal development of myelin might not have revealed a device to prevent regeneration of adult axons, but an important later developing mechanism - analogous to the repulsive guidance molecules present in early development — that

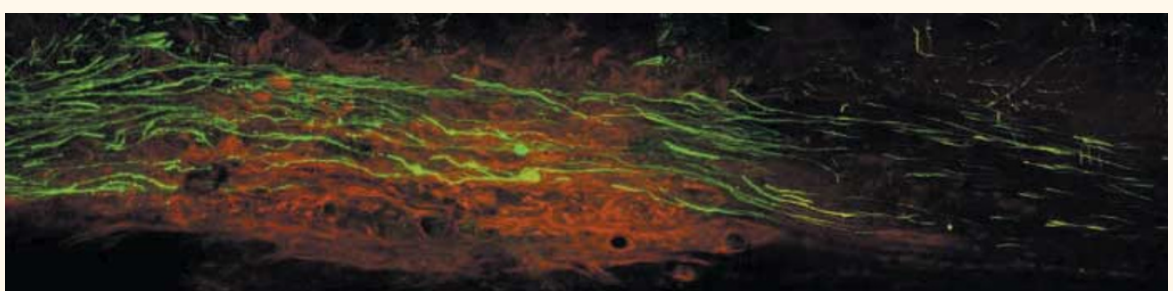

Figure 2 | Axon regeneration in the presence of olfactory ensheathing cells. Adult axons (green, biotin dextran label) regenerating through the distal tract after transplantation of olfactory ensheathing cells (p75 immunohistochemistry, red) into a complete corticospinal tract lesion. Reproduced, with permission, from REF. 19 ㄷ (1997) American Association for the Advancement of Science. 
a
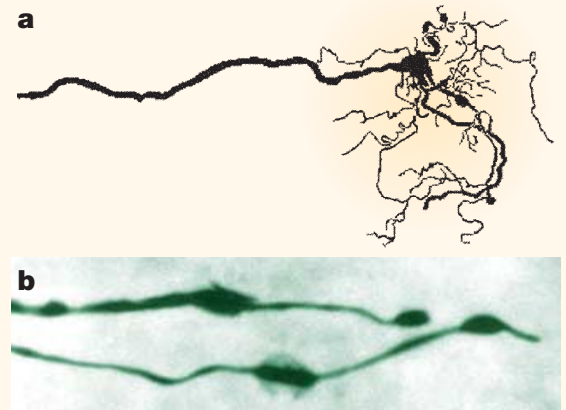

Figure 3 | Axon phenotypes during abortive and successful regeneration. a| Low power camera lucida drawing to show the profuse sprouting (so-called 'abortive regeneration') at the cut end of a single corticospinal tract axon at an unrepaired lesion site. Reproduced, with permission, from REF. 56 @ (1994) Society for Neuroscience. $\mathbf{b} \mid$ High power photomicrograph of two regenerating adult corticospinal axons showing the typical, unbranched form and simple tips of axons growing through a transplant of olfactory ensheathing cells. The axons are labelled with biotin dextran. Reproduced, with permission, from REF. 27 (c) (1998) Society for Neuroscience.

enables axons to grow down myelinated fibre tracts rapidly, efficiently and without branching (the so-called 'guard-rail' function ${ }^{38,39}$ ). According to this view, evolution did not put the repulsive molecules there to prevent growth, but rather to guide and facilitate it.

The failure of axonal regeneration that occurs in the early postnatal period has been ascribed to the onset of myelination ${ }^{40}$. There are, however, other possibilities, such as an age change in the inherent regenerative capacity of axons $\mathrm{s}^{41,42}$ and the maturation of astrocytic reactivity ${ }^{43-45}$. An obvious adaptive advantage of myelin is the acceleration of axonal excitation by saltatory conduction, which enables information to span the increasing distances of the adult central and peripheral nervous system without increasing the transmission time. But myelination might carry another advantage: if later developing axons are to follow the course of previously myelinated tracts (as occurs in the postnatal development of the corticospinal tracts ${ }^{46}$ ) the repulsive guidance channelling mechanism could serve to accelerate their growth over the longer distances that they have to cover as the brain and spinal cord become larger.

Growth promotion in adult fibre tracts. To understand how molecules function in the intact nervous system, it is necessary to take into account the detailed microstructure of the cellular environment in which the molecules are deployed. According to a view clearly enunciated by Crutcher, the reason for the difference between the effect of myelin inhibition in vitro and the situation in vivo is one of geometrical organization - "white matter supports parallel growth but inhibits nonparallel growth" ${ }^{37-49}$. In vitro, when the filopodia of a growth cone are faced with a mass of myelin molecules, there is no way through; the molecular mass is continuous, and the only choice for the growth cone is to collapse and/or to turn back or away. But when a growth cone is faced with a myelinated fibre tract in vivo, this is a structured environment, and the repulsive molecules are not diffusely scattered but are firmly anchored in the myelin sheaths. There is a way between the axons, however narrow.

One addition to Crutcher's model is the observation that in a tract that has not been subjected to direct damage, the way between these channels consists of fine longitudinal astrocytic processes ${ }^{20,24}$ (FIG. 4a,b). As the growing axon advances, it follows these longitudinal astrocytic processes, which guide it through the narrow channel, flanked on all sides by myelinated fibres that are bristling with repulsive molecules. The guidance mechanism provided by this massive surround of repulsion excludes all but forward motion, and is a necessary condition for the growth cone to advance. The value of such a mechanism is clear - it would confine growth to the straight and narrow trajectory between the existing myelinated axons. The growing axons must follow this narrow trajectory to reach their distant destination. In this way, the existing myelinated axons act as a guide for later growing axons in development and for regenerating axons in the adult.

The need to find and grow along the narrow astrocytic channels between existing myelinated axons explains why, when axons grow in adult myelinated tracts, they are dispersed rather than clustered (FIGS 1 and 2, and Y. Li et al., unpublished observations). The effect and purpose of the inhibitory molecules on the myelin sheaths is to constrain forward axon growth to the myelinated fibre tracts in the adult brain and spinal cord. Without them, axon elongation would be impossible. If this is true, inhibition of the myelin inhibitors will not enhance regeneration but prevent it.

The idea that myelin is actually facilitatory, and is probably essential for long-distance axonal growth in tracts is indicated by recent observations that axons can regenerate along partially denervated dorsal columns, in which a large proportion of the oligodendrocytes and their associated myelinated fibres are still present (REF. 50 and Y. Li et al., unpublished observations). However, if the dorsal columns are completely denervated, they become impenetrable to axons attempting to regenerate along them ${ }^{50}$. This is comparable to our findings of the failure of regeneration in the completely denervated optic nerve ${ }^{31}$. It implies that in a partially denervated tract, the surviving myelinated fibres are necessary to maintain the astroglial skeleton and to give direction to the regeneration by providing the channels lined by repulsive contact guidance, which are required for elongation of the regenerating axons along the aligned astrocytic tract processes. In the total absence of any myelinated fibres, the reactive astrocytes of the denervated tract simply condense down to form a dense and impenetrable glial scar (FIG. 4c), thereby eliminating all channels along which axons might grow.

From a therapeutic point of view, is there any way that inhibiting the myelin-associated repulsive mechanism might be beneficial? One possibility is that removing the mechanism that
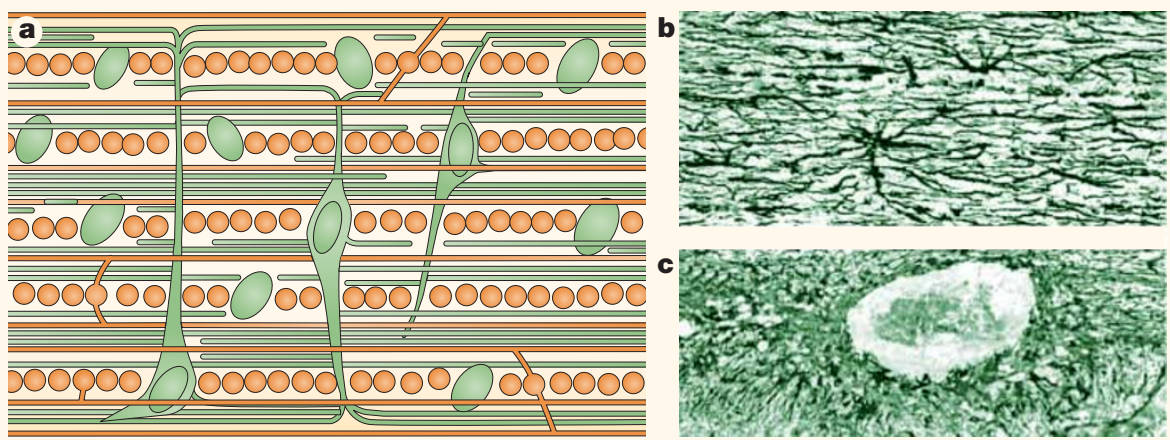

Figure 4 | Astrocytes in myelinated fibre tracts. a |A schematic representation to show that the glial skeleton of adult myelinated fibre tracts is made up of rows of cell bodies consisting of solitary astrocytes (green) and contiguous oligodendrocytes (orange) arranged in a fixed pattern, and giving rise to a longitudinal array of parallel astrocytic processes (green) and oligodendrocytic myelin (orange) ${ }^{24}$. b | Corticospinal tract showing the parallel array of astrocytic processes that are typical of adult myelinated fibre tracts. Astrocytes are immunostained with an antibody against glial fibrillary acidic protein (GFAP). c | The astrocytic hypertrophy and closing up of the longitudinal array of processes around a lesion in the corticospinal tract. Astrocytes are immunostained with an antibody against GFAP ${ }^{20}$ 
a
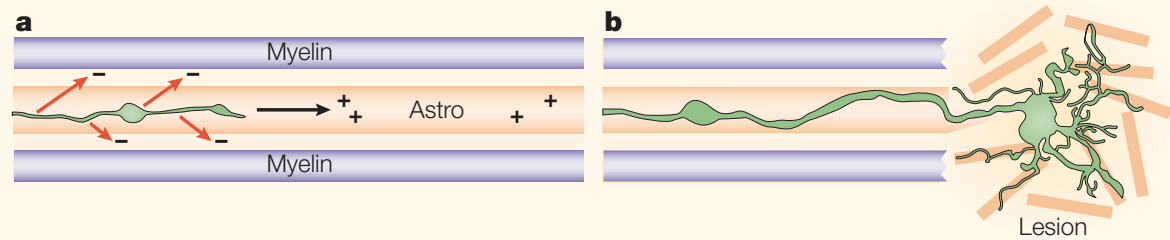

Figure 5 | Summary of the proposed mechanism for regulation of axon growth. a | In undamaged tracts, the growing tip of the axon (green) adheres to (+) and follows the direction of the underlying longitudinal astrocytic process (Astro). Its forward advance is kept on track and accelerated by the inhibition of side branching (red arrows) that is caused by contact with repellent molecules (-) on the surface of the surrounding myelinated axons. $\mathbf{b} \mid$ In the region of a lesion, oligodendrocytes and myelin are removed, but the proliferation of a meshwork of reactive astrocytic processes confronts the growing axon tips with an impenetrable tangle. The reason for the difference in the two situations is that the spatial organization of the membranes presenting the facilitatory (astrocytic) and inhibitory (oligodendrocytic) molecules to the axon tips is altered.

constrains axons to tracts could allow the surviving proximal segments of cut axons to express their innate growth potential and 'escape' from the tracts by branching and sending sprouts out into the neuropil ${ }^{51}$. These sprouts could then make short-range adventitious synaptic connections, which would allow functionally significant neural information to by-pass the lesion site and travel through multisynaptic relays to distant denervated sites and thereby ameliorate functional deficits. Inevitably, such sprouted pathways are abnormal, and in that sense, regeneration of the cut fibres along their original pathways would seem to be the reparative method of choice, as it holds out the possibility of restoring more normal patterns of connection. But the two approaches are not exclusive. Sprouting that is induced by temporary blockade of myelin-associated repulsive molecules, if carried out before or after measures to bring about regeneration of cut axons, could provide functionally valuable additions to the connections that are established by true regeneration.

\section{Conclusions}

The myelin inhibitory molecule theory ${ }^{1}$, initially based on the myelin-associated inhibitor Nogo, is now expanding to include other supposed myelin inhibitory molecules, such as Mag (myelin-associated glycoprotein) and Omgp (oligodendrocyte-myelin glycoprotein). On the basis of the prevailing dominance of the theory that inhibitory molecules prevent axon growth in white matter, it is becoming fashionable to postulate a parallel series of astrocyte-associated inhibitory molecules that prevent growth in grey matter ${ }^{23}$. The CNS is becoming conceived as a massive mountain of inhibition, quite at odds with the requirements of functional plasticity that is the fundamental basis of its adaptive advantage.
In this article, I have assembled some data for an alternative view (FIG. 5). First, as clearly enunciated by Aguayo $^{52}$, several different categories of adult central axons (although not necessarily all $^{53}$ ) retain an intrinsic power of growth, which they can express when provided with appropriate environmental stimuli, such as peripheral nerve or olfactory ensheathing cell grafts. Second, axons require an adhesive and supporting substrate on which to grow, and this is provided by the astrocytic processes that are present in white and grey matter. Third, the failure of regeneration in myelinated fibre tracts is due to a combination of molecular and configurational changes in the astrocytic pathways $\mathrm{s}^{14,54,55}$. Last, the purpose of the so-called inhibitory molecules that are associated with CNS myelin is to direct and facilitate long-distance growth of axons along myelinated tracts.

Geoffrey Raisman is at the Division of Neurobiology, The Norman and Sadie Lee Research Centre, National Institute for Medical Research, Medical Research Council, The Ridgeway, Mill Hill, London NW7 1AA, UK. e-mail:graisma@nimr.mrc.ac.uk. doi: $1038 / \mathrm{nrn} 132$

1. Filbin, M. T. Myelin-associated inhibitors of axonal regeneration in the adult mammalian CNS. Nature Rev. Neurosci. 4, 703-713 (2003).

2. McKerracher, L. \& Winton, M. J. Nogo on the go. Neuron 36, 345-348 (2002)

3. Schnell, L. \& Schwab, M. E. Axonal regeneration in the rat spinal cord produced by an antibody against myelinassociated neurite growth inhibitors. Nature $\mathbf{3 4 3}$ 269-272 (1990).

4. Schwab, M. E. \& Thoenen, H. Dissociated neurons regenerate into sciatic but not optic nerve explants in regenerate into sciatic but not optic nerve explants in culture irrespective of ne.

5. Crutcher, K. A. Tissue sections from the mature rat brain and spinal cord as substrates for neurite outgrowth in vitro: extensive growth on gray matter but little growth on white matter. Exp. Neurol. 104, 39-54 (1989).

6. Hunt, D. Coffin, R. S. \& Anderson, P. N. The Nogo receptor, its ligands and axonal regeneration in the spinal receptor, its ligands and axonal regeneration in the sp. Hunt, D., Coffin, R. S., Prinjha, R. K., Campbell, G. \& Hunt, D., Coffin, R. S., Prinjha, R. K., Campbell, G. \&
Anderson, P. N. Nogo-A expression in the intact and Anderson, P. N. Nogo-A expression in the intact and
injured nervous system. Mol. Cell. Neurosci. (in the press).
8. Hunt, D., Mason, M. R., Campbell, G., Coffin, R. \& Anderson, P. N. Nogo receptor mRNA expression in And Neurosci. 20,537-552 (2002).

9. Huang, D. W., McKerracher, L., Braun,P. E. \& David, S. A therapeutic vaccine approach to stimulate axon regeneration in the adult mammalian spinal cord. Neuron 24, 639-647 (1999).

10. Kim, J. E., Li, S. X., GrandPré, T., Qiu, D. \& Strittmatter, S. $M$. Axon regeneration in young adult mice lacking NogoA/B. Neuron 38, 187-199 (2003).

11. Woolf, C. J. No Nogo: now where to go? Neuron $\mathbf{3 8}$ 153-156 (2003)

12. Zheng, B. H. et al. Lack of enhanced spinal regeneration in Nogo-deficient mice. Neuron 38, 213-224 (2003).

13. Simonen, M. et al. Systemic deletion of the myelinassociated outgrowth inhibitor Nogo-A improves regenerative and plastic responses after spinal cord injury. Neuron 38, 201-211 (2003).

14. Davies, S. J. A. et al. Regeneration of adult axons in white matter tracts of the central nervous system. Nature $\mathbf{3 9 0}$ 680-683 (1997)

15. Wictorin, K., Brundin, P., Sauer, H., Lindvall, O. \& Björklund, A. Long distance directed axonal growth from human dopaminergic mesencephalic neuroblasts implanted along the nigrostriatal pathway in 6-hydroxydopamine lesioned adult rats. J. Comp. Neurol. 323, 475-494 (1992).

16. Davies, S. J. A. \& Silver, J. Adult axon regeneration in adult CNS white matter. Trends Neurosci. 21, 515 (1998).

17. Davies, S. J. A., Field, P. M. \& Raisman, G. Long fibre growth by axons of embryonic mouse hippocampal neurons micro-transplanted into the adult rat fimbria. Eur. J. Neurosci. 5, 95-106 (1993).

18. Li, Y. \& Raisman, G. Long axon growth from embryonic neurons transplanted into myelinated tracts of the adult rat spinal cord. Brain Res. 629, 115-127 (1993).

19. Li, Y., Field, P. M. \& Raisman, G. Repair of adult rat corticospinal tract by transplants of olfactory ensheathing cells. Science 277, 2000-2002 (1997).

20. Li, Y. \& Raisman, G. Sprouts from cut corticospinal axons persist in the presence of astrocytic scarring in long-term esions of the adult rat spinal cord. Exp. Neurol. 134 102-111 (1995).

21. Bradbury, E. J. et al. Chondroitinase ABC promotes functional recovery after spinal cord injury. Nature $\mathbf{4 1 6}$, 636-640 (2002).

22. Grimpe, B. \& Silver, J. The extracellular matrix in axon regeneration. Prog. Brain Res. 137, 333-349 (2002)

23. Silver, J. \& Miller, J. H. Regeneration beyond the glial scar. Nature Rev. Neurosci. 5, 146-156 (2004).

24. Suzuki, M. \& Raisman, G. The glial framework of centra white matter tracts: segmented rows of contiguous interfascicular oligodendrocytes and solitary astrocytes give rise to a continuous meshwork of transverse and longitudinal processes in the adult rat fimbria. Glia 6 . 222-235 (1992)

25. Meier, C. \& Sollmann, H. Glial outgrowth and central-type myelination of regenerating axons in spinal nerve roots following transection and suture: light and electron microscopic study in the pig. Neuropathol. appl. Neurobiol. 4, 21-35 (1978)

26. Houlé, J. The structural integrity of glial scar tissue associated with a chronic spinal cord lesion can be altered by transplanted fetal spinal cord tissue. J. Neurosci. Res. 31, 120-130 (1992).

27. Li, Y., Field, P. M. \& Raisman, G. Regeneration of adult rat corticospinal axons induced by transplanted olfactory ensheathing cells. J. Neurosci. 18, 10514-10524 (1998)

28. McDonald, J. W. Althomsons, S. P., Hyrc, K. L., Choi, D. W. \& Goldberg, M. P. Oligodendrocytes from forebrain are highly vulnerable to AMPA/kainate receptor-mediated excitotoxicity. Nature Med. 4, 291-297 (1998).

29. Li, Y., Field, P. M. \& Raisman, G. Death of oligodendrocytes and microglial phagocytosis of myelin precede immigration of Schwann cells into the spinal cord. J. Neurocytol. 28, 417-427 (1999).

30. Warden, P. et al. Delayed glial cell death following Wallerian degeneration in white matter tracts after spinal cord dorsal column cordotomy in adult rats. Exp. Neurol. 168, 213-224 (2001).

31. Li, Y., Sauvé, Y., Li, D., Lund, R. D. \& Raisman, G. Transplanted olfactory ensheathing cells promote regeneration of cut adult rat optic nerve axons. J. Neurosci. 23, 7922-7930 (2003).

32. Fawcett, J. W., Housden, E., Smith-Thomas, L. \& Meyer, R. L. The growth of axons in three-dimensional astrocyte cultures. Dev. Biol. 135, 449-458 (1989).

33. Baird, D. H., Hatten, M. E. \& Mason, C. A. Cerebellar target neurons provide a stop signal for afferent neurite extension in vitro. J. Neurosci. 12, 619-634 (1992). 
34. Bovolenta, P. \& Mason, C. Growth cone morphology varies with position in the developing mouse visual pathway from retina to first targets. J. Neurosci. 7 , 1447-1460 (1987)

35. Tessier-Lavigne, M. \& Goodman, C. S. The molecula biology of axon guidance. Science $\mathbf{2 7 4}, 1123-1133$ (1996)

36. Araújo, S. J. \& Tear, G. Axon guidance mechanisms and molecules: lessons from invertebrates. Nature Rev. Neurosci. 4, 910-922 (2003).

37. Hopker, V. H., Shewan, D., Tessier-Lavigne, M., Poo, M. \& Holt, C. Growth-cone attraction to netrin-1 is converted to repulsion by laminin-1. Nature 401, 69-73 (1999).

38. Schwab, M. E., Kapfhammer, J. P. \& Bandtlow, C. E. Inhibitors of neurite growth. Annu. Rev. Neurosci. 16, 565-595 (1993).

39. Schwab, M. E. \& Schnell, L. Channeling of developing rat corticospinal tract axons by myelin-associated neurite growth inhibitors. Jeurosci 11,709-721 (1091).

40. Vanek, P., Thallmair, M., Schwab, M. E. \& Kapfhammer, J. P. Increased lesion-induced sprouting of corticospinal fibres in the myelin-free rat spinal cord. Eur. J. Neurosci. 10, 45-56 (1998)

41. Li, D., Field, P. M. \& Raisman, G. Failure of axon regeneration in postnatal rat entorhino-hippocampal slice co-culture is due to maturation of the axon, not that of the

Sci. 7, 1164-1171 (1995) I. The developmental loss of the ability of Purkinje cells to regenerate their axons occurs in the absence of myelin: an in vitro model to prevent myelination. J. Neurosci. $\mathbf{2 3}$ 8318-8329 (2003).
43. Maxwell, W. L., Follows, R., Ashhurst, D. E. \& Berry, M. The response of the cerebral hemisphere of the rat to injury. II. The neonatal rat. Philos. Trans. R. Soc. Lond. B 328, 501-513 (1990).

44. Maxwell, W. L., Follows, R., Ashhurst, D. E. \& Berry, M The response of the cerebral hemisphere of the rat to injury. I. The mature rat. Philos. Trans. R. Soc. Lond. B 328, 479-500 (1990).

45. Kliot, M., Smith, G. M., Siegal, J. D. \& Silver, J. Astrocytepolymer implants promote regeneration of dorsal root foly into the adut mammalian spinal cord. Exp. Net 109, 57-69 (1990).

46. De Kort, E. J. M., Gribnau, A. A. M., Van Aanholt, H. T. H \& Nieuwenhuys, R. On the development of the pyramidal tract in the rat. I. The morphology of the growth zone. Anat. Embryol. 172, 195-204 1985).

47. Pettigrew, D. B. \& Crutcher, K. A. White matter of the CNS supports or inhibits neurite outgrowth in vitro depending on geometry. J. Neurosci. 19, 8358-8366 (1999).

48. Pettigrew, D. B. \& Crutcher, K. A. Myelin contributes to the parallel orientation of axonal growth on white matter in vitro. BMC Neurosci. 2, 9 (2001).

49. Pettigrew, D. B., Shockley, K. P. \& Crutcher, K. A. Disruption of spinal cord white matter and sciatic nerve geometry inhibits axonal growth in vitro in the absence of glial scarring. BMC Neurosci. 2, 8 (2001).

50. Ramer, M. S., Duraisingam, I., Priestley, J. V. \& McMahon, S. B. Two-tiered inhibition of axon regeneration at the dorsal root entry zone. J. Neurosci. 21, 2651-2660 (2001)
51. Raineteau, O. \& Schwab, M. E. Plasticity of motor systems after incomplete spinal cord injury. Nature Rev. Neurosci. 2, 263-273 (2001).

52. David, S. \& Aguayo, A. J. Axonal elongation into peripheral nervous system 'bridges' after central nervous system injury in adult rats. Science 214, 931-933 (1981).

53. Rossi, F., Jankovski, A. \& Sotelo, C. Differential regenerative response of Purkinje cell and inferior olivary axons confronted with embryonic grafts: environmental cues versus intrinsic neuronal determinants. J. Comp. Neurol. 359, 663-677 (1995).

54. Davies, S. J. A., Field, P. M. \& Raisman, G. Regeneration of cut adult axons fails even in the presence of continuous aligned glial pathways. Exp. Neurol. 142 203-216 (1996)

55. Davies, S. J. A. et al. Robust adult axon regeneration in central nervous system white matter tracts. Soc. Neurosci. Abstr. 23, 1448 (1997).

56. Li, Y. \& Raisman, G. Schwann cells induce sprouting in motor and sensory axons in the adult rat spinal cord. J. Neurosci. 14, 4050-4063 (1994).

Competing interests statement The author declares that he has no competing financial interests.

\section{(D) Online links}

\section{FURTHER INFORMATION}

The Raisman group's homepage:

http://www.nimr.mrc.ac.uk/neurobiol/raisman/

Access to this interactive links box is free online. 